\title{
Exponentially suppressed cosmological constant and enhanced gauge symmetry in interpolating heterotic models
}

\author{
Sota Nakajima* \\ Department of Mathematics and Physics, Graduate School of Science, \\ Osaka City University \\ E-mail: Sotanakadsci.osaka-cu.ac.jp
}

\begin{abstract}
We construct 9D heterotic string models deformed by one Wilson line which interpolate from $10 \mathrm{D}$ supersymmetric string models to the $10 \mathrm{D}$ non-supersymmetric $S O(16) \times S O(16)$ model, and calculate the cosmological constants in the large radius region which depend on the Wilson line. We find the configuration of the Wilson line on which the gauge symmetry is enhanced to $S O(18) \times S O(14)$ and the cosmological constant is exponentially suppressed. We discuss the stability of the Wilson line from the one-loop effective potential.
\end{abstract}

Corfu Summer Institute 2019 "School and Workshops on Elementary Particle Physics and Gravity" (CORFU2019)

31 August - 25 September 2019

Corfù, Greece

\footnotetext{
* Speaker.
} 


\section{Introduction}

String theory can give the descriptions of all the observed matters and interactions including gravity and has been explored to answer a lot of questions about fundamental physics. For theoretical and phenomenological reasons, most works focused on string theories with spacetime supersymmetry (SUSY) and compactified them, preserving SUSY by Calabi-Yau manifolds or orbifolds [1-3]. However, since the LHC experiment suggests that there is no SUSY in the multi $\mathrm{TeV}$ scale, the scenario that SUSY is already broken at very high energy such as string/Planck scale has been brought to attention. In particular, the constructions of realistic models such as the Standard(-like) Model directly from non-supersymmetric string theories [4,5] have been studied. Although it is natural to consider non-supersymmetric string phenomenology, we will be faced with some difficulties in such top-down approaches. One of them is the problem of vacuum instabilities causing by non-vanishing dilaton tadpoles. In general, the vacuum energies (the cosmological constants) give a nonzero value without SUSY since there are no cancellations between fermionic and bosonic states at each energy level. Consequently, the dilaton tadpoles, which are proportional to the cosmological constants, are non-vanishing and give rise to the serious vacuum instabilities. In order to discuss non-supersymmetric string phenomenology, therefore, the good starting points are non-supersymmetric string models whose cosmological constants are sufficiently small.

While some constructions of non-supersymmetric string models with small or zero cosmological constants have been proposed, we adopt, in this talk, the interpolating models [6] as a clue to fix the problem of the vacuum instabilities. The interpolating models are constructed by the coordinate dependent compactification (CDC) $[7,8]$ which is the stringy version of the Scherk-Schwartz compactification [9], and the partition function of 9D interpolating models is, in general, written as the following form [10]:

$$
\begin{aligned}
Z_{\mathrm{int}}^{(9)}=\frac{1}{2} & Z_{B}^{(7)}\left\{\Lambda_{0,0}\left(Z_{+}^{+}+Z_{-}^{+}\right)+\Lambda_{1 / 2,0}\left(Z_{+}^{+}-Z_{-}^{+}\right)\right. \\
& \left.+\Lambda_{0,1 / 2}\left(Z_{+}^{-}+Z_{-}^{-}\right)+\Lambda_{1 / 2,1 / 2}\left(Z_{+}^{-}-Z_{-}^{-}\right)\right\}
\end{aligned}
$$

where $Z_{B}^{(N)}=\tau_{2}^{-N / 2}(\eta \bar{\eta})^{-N}$ is the contributions from bosonic string coordinates in spacetime dimensions, $Z_{ \pm}^{ \pm}$is those from fermionic string coordinates ${ }^{1}$, and $\Lambda_{\alpha, \beta}$ is the momentum lattice defined as

$$
\Lambda_{\alpha, \beta}=(\eta \bar{\eta})^{-1} \sum_{n \in 2(\boldsymbol{Z}+\alpha)} \sum_{w \in \boldsymbol{Z}+\beta} q^{\frac{\alpha^{\prime}}{2} p_{L}^{2}} \bar{q}^{\frac{\alpha^{\prime}}{2} p_{R}^{2}}=(\eta \bar{\eta})^{-1} \sum_{n \in 2(\boldsymbol{Z}+\alpha)} \sum_{w \in \boldsymbol{Z}+\beta} q^{\frac{1}{4}(n a+w / a)^{2}} \bar{q}^{\frac{1}{4}(n a-w / a)^{2}}
$$

with a dimensionless inverse radius $a=\sqrt{\alpha^{\prime}} / R$. In order for $Z_{\text {int }}^{(9)}$ to be modular invariant, the following relations must be satisfied:

$$
Z_{+}^{+}(\tau)=Z_{+}^{+}(-1 / \tau), \quad Z_{-}^{-}(\tau)=Z_{-}^{-}(-1 / \tau), \quad Z_{+}^{-}(\tau)=Z_{-}^{+}(-1 / \tau) .
$$

We can check that the behaviors of $\Lambda_{\alpha, \beta}$ in the limits $a \rightarrow 0$ and $a \rightarrow \infty$ are respectively

$$
\Lambda_{\alpha, \beta} \rightarrow \frac{1}{2 a} Z_{B}^{(1)} \delta_{\beta, 0}, \quad \Lambda_{\alpha, \beta} \rightarrow a Z_{B}^{(1)} \delta_{\alpha, 0} .
$$

\footnotetext{
${ }^{1}$ In heterotic strings, $Z_{ \pm}^{ \pm}$contains rank 16 current algebras. But, they can be regarded as fermionic string coordinates by fermionization.
} 
Then, we obtain, in each limit, the two endpoint 10D models $M_{1}$ and $M_{2}$ whose partition functions are written as respectively

$$
Z_{M_{1}}=Z_{B}^{(8)} Z_{+}^{+}, \quad Z_{M_{2}}=\frac{1}{2} Z_{B}^{(8)}\left(Z_{+}^{+}+Z_{-}^{+}+Z_{+}^{-}+Z_{-}^{-}\right) .
$$

While model $M_{1}$ and $M_{2}$ are related discretely by a $Z_{2}$ twist $Q: Z_{+}^{ \pm} \rightarrow Z_{-}^{ \pm}$, they can be connected continuously through $Z_{\text {int }}^{(9)}$ with a continuous parameter $a$. In this sense, 9D string models whose partition functions are written as $(\mathbb{L})$ interpolate between two different 10D string models. In particular, if model $M_{1}$ is supersymmetric and model $M_{2}$ is non-supersymmetric, then the cosmological constant of the interpolating model at one-loop level can be written as $[6,11]$

$$
\Lambda^{(9)}=\left(n_{F}-n_{B}\right) \xi a^{9}+\mathscr{O}\left(e^{-1 / a}\right), \quad a \approx 0
$$

where $n_{F}$ and $n_{B}$ are the degrees of freedom of massless fermions and massless bosons respectively. Namely, in string models that interpolate from supersymmetric models to non-supersymmetric models, the cosmological constants are exponentially suppressed in the region where SUSY is asymptotically restoring if $n_{F}=n_{B}$. Such models are referred to as super no-scale models and preferred in order to investigate non-supersymmetric string phenomenology [12-22].

Interpolating models can be deformed by turning on constant backgrounds because they are constructed by compactifying higher-dimensional models. Heterotic string models $d$-dimensional compactified have the freedom of the deformations which are represented by the coset

$$
\frac{S O(16+d, d)}{S O(16+d) \times S O(d)}
$$

These deformations are realized by adding the following terms to the world-sheet action $[23,24]$ :

$$
A_{I i} \int d^{2} z \partial X_{L}^{I} \bar{\partial} X_{R}^{i}+C_{j i} \int d^{2} z \partial X_{L}^{j} \bar{\partial} X_{R}^{i}
$$

for $I=1, \cdots, 16, i, j=10-d, \cdots, 9$, where the constant $A_{I i}$ corresponds to the background gauge fields (Wilson lines) and the constant $C_{j i}$ is decomposed into metric $g_{j i}$ and antisymmetric tensor $B_{j i}$. In this talk, we set, for simplicity, $A_{1}^{9}=A$ and $A_{I \neq 1}^{9}=0$, and consider 9D interpolating models deformed by one Wilson line. For detail of this proceeding, please refer to Ref. [25]. The 9D interpolating models deformed by the complete set of Wilson lines and the moduli space are studied in Ref. [26], which is our current work.

\section{Two examples of interpolations}

In this talk, we focus on two interpolations between the following 10D string models:

- Model I: SUSY $S O(32)$ model $\longleftrightarrow S O(16) \times S O(16)$ model

- Model II: SUSY $E_{8} \times E_{8}$ model $\longleftrightarrow S O(16) \times S O(16)$ model 
In Model I, the partition functions of the endpoint models $M_{1}$ and $M_{2}$ are respectively written as follows:

$$
\begin{aligned}
Z_{M_{1}}=Z_{B}^{(8)} & \left(\bar{V}_{8}-\bar{S}_{8}\right)\left(O_{16} O_{16}+V_{16} V_{16}+S_{16} S_{16}+C_{16} C_{16}\right), \\
Z_{M_{2}}=Z_{B}^{(8)} & \left\{\bar{O}_{8}\left(V_{16} C_{16}+C_{16} V_{16}\right)+\bar{V}_{8}\left(O_{16} O_{16}+S_{16} S_{16}\right)\right. \\
& \left.-\bar{S}_{8}\left(V_{16} V_{16}+C_{16} C_{16}\right)-\bar{C}_{8}\left(O_{16} S_{16}+S_{16} O_{16}\right)\right\}
\end{aligned}
$$

where $\left(O_{2 n}, V_{2 n}, S_{2 n}, C_{2 n}\right)$ are the $S O(2 n)$ characters defined as

$$
\begin{aligned}
& \left(\begin{array}{l}
O_{2 n} \\
V_{2 n}
\end{array}\right) \equiv \frac{1}{2 \eta^{n}}\left(\vartheta^{n}\left[\begin{array}{l}
0 \\
0
\end{array}\right](0, \tau) \pm \vartheta^{n}\left[\begin{array}{c}
0 \\
1 / 2
\end{array}\right](0, \tau)\right) \\
& \left(\begin{array}{l}
S_{2 n} \\
C_{2 n}
\end{array}\right) \equiv \frac{1}{2 \eta^{n}}\left(\vartheta^{n}\left[\begin{array}{c}
1 / 2 \\
0
\end{array}\right](0, \tau) \pm \vartheta^{n}\left[\begin{array}{l}
1 / 2 \\
1 / 2
\end{array}\right](0, \tau)\right) .
\end{aligned}
$$

Note that the products of the theta functions in the above characters imply the sums over the zero modes of internal bosonic string coordinates from the viewpoint of bosonization. According to (ㅁ. $)$, the partition function of Model I is

$$
\begin{aligned}
Z_{\text {int }}^{(9)}=Z_{B}^{(7)} & \left\{\Lambda_{0,0}\left(\bar{V}_{8}\left(O_{16} O_{16}+S_{16} S_{16}\right)-\bar{S}_{8}\left(V_{16} V_{16}+C_{16} C_{16}\right)\right)\right. \\
& +\Lambda_{0,1 / 2}\left(\bar{O}_{8}\left(V_{16} C_{16}+C_{16} V_{16}\right)-\bar{C}_{8}\left(O_{16} S_{16}+S_{16} O_{16}\right)\right) \\
& +\Lambda_{1 / 2,0}\left(\bar{V}_{8}\left(V_{16} V_{16}+C_{16} C_{16}\right)-\bar{S}_{8}\left(O_{16} O_{16}+S_{16} S_{16}\right)\right) \\
& \left.+\Lambda_{1 / 2,1 / 2}\left(\bar{O}_{8}\left(O_{16} S_{16}+S_{16} O_{16}\right)-\bar{C}_{8}\left(V_{16} C_{16}+C_{16} V_{16}\right)\right)\right\} .
\end{aligned}
$$

We can obtain the spectrum at the mass level $N$ by expanding the partition function in $q$ and figuring out the coefficients of $(q \bar{q})^{N}$. For example, the massless spectrum of Model I is

- the nine-dimensional gravity multiplet: graviton $G_{\mu v}$, anti-symmetric tensor $B_{\mu v}$, dilaton $\phi$;

- the gauge bosons transforming in the adjoint representation of $S O(16) \times S O(16) \times U(1)^{2}$;

- a spinor transforming in the $(16,16)$ of $S O(16) \times S O(16)$.

Note that the Abelian factors $U(1)^{2}$ are generated by $G_{\mu 9}$ and $B_{\mu 9}$. In Model I, therefore, $n_{F}-n_{B}=$ 64, which means that the cosmological constant (ㅁ.6) is positive in the small- $a$ region.

In Model II, the partition function of model $M_{2}$ is the same as in Model I, and that of model $M_{1}$ is

$$
Z_{M_{1}}=Z_{B}^{(8)} Z_{+}^{+}=Z_{B}^{(8)}\left(\bar{V}_{8}-\bar{S}_{8}\right)\left(O_{16}+S_{16}\right)\left(O_{16}+S_{16}\right) .
$$

Then, the partition function of this interpolating model is

$$
\begin{aligned}
Z_{\text {int }}^{(9)}=Z_{B}^{(7)} & \left\{\Lambda_{0,0}\left(\bar{V}_{8}\left(O_{16} O_{16}+S_{16} S_{16}\right)-\bar{S}_{8}\left(O_{16} S_{16}+S_{16} O_{16}\right)\right)\right. \\
& +\Lambda_{1 / 2,0}\left(\bar{V}_{8}\left(O_{16} S_{16}+S_{16} O_{16}\right)-\bar{S}_{8}\left(O_{16} O_{16}+S_{16} S_{16}\right)\right) \\
& +\Lambda_{0,1 / 2}\left(\bar{O}_{8}\left(V_{16} C_{16}+C_{16} V_{16}\right)-\bar{C}_{8}\left(V_{16} V_{16}+C_{16} C_{16}\right)\right) \\
& \left.+\Lambda_{1 / 2,1 / 2}\left(\bar{O}_{8}\left(V_{16} V_{16}+C_{16} C_{16}\right)-\bar{C}_{8}\left(V_{16} C_{16}+C_{16} V_{16}\right)\right)\right\} .
\end{aligned}
$$

The massless spectrum of this model is 
- the nine-dimensional gravity multiplet: graviton $G_{\mu \nu}$, anti-symmetric tensor $B_{\mu \nu}$, dilaton $\phi$;

- the gauge bosons transforming in the adjoint representation of $S O(16) \times S O(16) \times U(1)^{2}$;

- a spinor transforming in the $(128,1) \oplus(1,128)$ of $S O(16) \times S O(16)$.

In Model II, $n_{F}-n_{B}=64$ as well as in Model I.

We can obtain the additional massless states if the parameter $a$ is adjusted appropriately. There are, however, no values of $a$ such that $n_{F}-n_{B}=0$, in both interpolating models.

\section{Deformation by one Wilson line}

Let us deform the interpolating models by adding the following term to the world-sheet action:

$$
A \int d^{2} z \partial X_{L}^{I=1} \bar{\partial} X_{R}^{i=9}
$$

This term only affects the quantization of string zero modes since it is a total derivative. The leftand right-moving internal momenta $\ell_{L}\left(=\ell_{L}^{I=1}\right), p_{L}\left(=p_{L}^{i=9}\right)$ and $p_{R}\left(=p_{R}^{i=9}\right)$ are boosted as follows:

$$
\begin{aligned}
& \ell_{L}^{\prime}=\frac{1}{\sqrt{2 \alpha^{\prime}}}\left(\sqrt{2} m-2 A \frac{w}{a_{0}}\right), \\
& p_{L}^{\prime}=\frac{1}{\sqrt{2 \alpha^{\prime}}}\left(\sqrt{2} A m+a_{0} n+\left(1-A^{2}\right) \frac{w}{a_{0}}\right), \\
& p_{L}^{\prime}=\frac{1}{\sqrt{2 \alpha^{\prime}}}\left(\sqrt{2} A m+a_{0} n-\left(1+A^{2}\right) \frac{w}{a_{0}}\right),
\end{aligned}
$$

where $a_{0} \equiv \sqrt{1+A^{2}} a$. It is convenient to introduce a complex parameter $t$ defined as

$$
t=t_{1}+i t_{2}=\frac{1}{\sqrt{2}} A a_{0}^{-1}+\frac{i}{\sqrt{2}} a_{0}^{-1} .
$$

Then, the above momenta are rewritten as

$$
\begin{aligned}
& \ell_{L}^{\prime}=\frac{1}{\sqrt{\alpha^{\prime}}}\left(m-2 t_{1} w\right), \\
& p_{L}^{\prime}=\frac{1}{\sqrt{\alpha^{\prime}}} t_{2}^{-1}\left(t_{1} m+\frac{n}{2}-\left(t_{1}^{2}-t_{2}^{2}\right) w\right), \\
& p_{R}^{\prime}=\frac{1}{\sqrt{\alpha^{\prime}}} t_{2}^{-1}\left(t_{1} m+\frac{n}{2}-\left(t_{1}^{2}+t_{2}^{2}\right) w\right) .
\end{aligned}
$$

In the partition function, the momentum lattice and a theta function in one of the two left-moving $S O(16)$ characters are convoluted as follows:

$$
\Lambda_{\alpha, \beta}\left(\frac{\vartheta\left[\begin{array}{l}
\gamma \\
\delta
\end{array}\right]}{\eta}\right)^{8} \rightarrow \Lambda_{(\gamma, \delta)}^{(\alpha, \beta)}\left(t_{1}, t_{2}\right)\left(\frac{\vartheta\left[\begin{array}{l}
\gamma \\
\delta
\end{array}\right]}{\eta}\right)^{7}
$$


where $\Lambda_{(\gamma, \delta)}^{(\alpha, \beta)}$ is a $S O(2,1)$ momentum lattice defined as

$$
\Lambda_{(\gamma, \delta)}^{(\alpha, \beta)}\left(t_{1}, t_{2}\right) \equiv(\eta \bar{\eta})^{-1} \eta^{-1} \sum_{n, w, m}(-1)^{2 m \delta} q^{\frac{\alpha^{\prime}}{2}\left(p_{L}^{\prime 2}+\ell_{L}^{\prime 2}\right)} \bar{q}^{\frac{\alpha^{\prime}}{2} p_{R}^{\prime 2}}
$$

for $n \in \mathbf{Z}+\alpha, w \in 2(\boldsymbol{Z}+\beta), m \in \mathbf{Z}+\gamma$. We can check that the lattice $\Lambda_{(\gamma, \boldsymbol{\delta})}^{(\alpha, \beta)}$ is invariant under the shift

$$
t \rightarrow t+2
$$

Therefore, the fundamental region of the moduli space is

$$
-1<t_{1} \leq 1, \quad t_{2} \geq 0
$$

\subsection{Model I with one Wilson line}

Applying (B.9) to ([2.5), the partition function of Model I deformed by the Wilson line is

$$
\begin{aligned}
Z_{\mathrm{int}}^{(9)}\left(t_{1}, t_{2}\right)= & Z_{B}^{(7)}\left\{\bar{V}_{8}\left(O_{16}^{(0,0)} O_{16}+S_{16}^{(0,0)} S_{16}\right)-\bar{S}_{8}\left(V_{16}^{(0,0)} V_{16}+C_{16}^{(0,0)} C_{16}\right)\right. \\
& +\bar{V}_{8}\left(V_{16}^{(1 / 2,0)} V_{16}+C_{16}^{(1 / 2,0)} C_{16}\right)-\bar{S}_{8}\left(O_{16}^{(1 / 2,0)} O_{16}+S_{16}^{(1 / 2,0)} S_{16}\right) \\
& +\bar{O}_{8}\left(V_{16}^{(0,1 / 2)} C_{16}+C_{16}^{(0,1 / 2)} V_{16}\right)-\bar{C}_{8}\left(O_{16}^{(0,1 / 2)} S_{16}+S_{16}^{(0,1 / 2)} O_{16}\right) \\
& \left.+\bar{O}_{8}\left(O_{16}^{(1 / 2,1 / 2)} S_{16}+S_{16}^{(1 / 2,1 / 2)} O_{16}\right)-\bar{C}_{8}\left(V_{16}^{(1 / 2,1 / 2)} C_{16}+C_{16}^{(1 / 2,1 / 2)} V_{16}\right)\right\},
\end{aligned}
$$

where $\left(O_{2 n}^{(\alpha, \beta)}, V_{2 n}^{(\alpha, \beta)}, S_{2 n}^{(\alpha, \beta)}, C_{2 n}^{(\alpha, \beta)}\right)$ are defined by

$$
\begin{aligned}
& \left(\begin{array}{c}
O_{2 n}^{(\alpha, \beta)} \\
V_{2 n}^{(\alpha, \beta)}
\end{array}\right) \equiv \frac{1}{2 \eta^{n-1}}\left(\Lambda_{(0,0)}^{(\alpha, \beta)} \vartheta^{n-1}\left[\begin{array}{l}
0 \\
0
\end{array}\right](0, \tau) \pm \Lambda_{(0,1 / 2)}^{(\alpha, \beta)} \vartheta^{n-1}\left[\begin{array}{c}
0 \\
1 / 2
\end{array}\right](0, \tau)\right) \\
& \left(\begin{array}{c}
S_{2 n}^{(\alpha, \beta)} \\
C_{2 n}^{(\alpha, \beta)}
\end{array}\right) \equiv \frac{1}{2 \eta^{n-1}}\left(\Lambda_{(1 / 2,0)}^{(\alpha, \beta)} \vartheta^{n-1}\left[\begin{array}{c}
1 / 2 \\
0
\end{array}\right](0, \tau) \pm \Lambda_{(1 / 2,1 / 2)}^{(\alpha, \beta)} \vartheta^{n-1}\left[\begin{array}{c}
1 / 2 \\
1 / 2
\end{array}\right](0, \tau)\right)
\end{aligned}
$$

Let us see the massless spectrum of this model. At generic points in the moduli space, the massless spectrum is

- the nine-dimensional gravity multiplet: graviton $G_{\mu v}$, anti-symmetric tensor $B_{\mu v}$, dilaton $\phi$;

- the gauge bosons transforming in the adjoint representation of $S O(16) \times S O(14) \times U(1)^{3}$;

- a spinor transforming in the $(16,14)$ of $S O(16) \times S O(14)$.

Note that the gauge symmetry is partially broken because of the deformation by the Wilson line. We can find special points in the moduli space where the additional massless states appear. In this talk, we assume that $t_{2}$ is fixed at a large value such that the formula (ㅁ.6) is valid and consider the one-dimensional moduli space characterized by $t_{1}$. At the points $t_{1}=0,1$, the following massless states are found in addition to the above massless states:

- two vectors transforming in the $(1,14)$ of $S O(16) \times S O(14)$; 
- two spinors transforming in the $(\mathbf{1 6}, \mathbf{1})$ of $S O(16) \times S O(14)$.

As a result, on these points, the gauge symmetry is enhanced to $S O(16) \times S O(16)^{2}$ and a spinor transforming in the $(\mathbf{1 6}, \mathbf{1 6})$ of $S O(16) \times S O(16)$ becomes massless. Note that the massless spectrum at the points is the same as in the no Wilson line case in section 1 . The other special points in the moduli space are $t_{1}= \pm 1 / 2$, where the following states become massless:

- two vectors transforming in the $(\mathbf{1 6}, 1)$ of $S O(16) \times S O(14)$;

- two spinors transforming in the $(\mathbf{1}, 14)$ of $S O(16) \times S O(14)$.

At these points, therefore, the gauge symmetry is enhanced to $S O(18) \times S O(14)$ and a massless spinor transforms in the $(\mathbf{1 8}, \mathbf{1 4})$ of $S O(18) \times S O(14)$. Note that $n_{F}-n_{B}=0$ at these points, which means that the cosmological constant is exponentially suppressed.

\subsection{Model II with one Wilson line}

The partition function of Model II deformed by the Wilson line is

$$
\begin{aligned}
Z_{\mathrm{int}}^{(9)}= & Z_{B}^{(7)}\left\{\bar{V}_{8}\left(O_{16}^{(0,0)} O_{16}+S_{16}^{(0,0)} S_{16}\right)-\bar{S}_{8}\left(O_{16}^{(0,0)} S_{16}+S_{16}^{(0,0)} O_{16}\right)\right. \\
& +\bar{V}_{8}\left(O_{16}^{(1 / 2,0)} S_{16}+S_{16}^{(1 / 2,0)} O_{16}\right)-\bar{S}_{8}\left(O_{16}^{(1 / 2,0)} O_{16}+S_{16}^{(1 / 2,0)} S_{16}\right) \\
& +\bar{O}_{8}\left(V_{16}^{(0,1 / 2)} C_{16}+C_{16}^{(0,1 / 2)} V_{16}\right)-\bar{C}_{8}\left(V_{16}^{(0,1 / 2)} V_{16}+C_{16}^{(0,1 / 2)} C_{16}\right) \\
& \left.+\bar{O}_{8}\left(V_{16}^{(1 / 2,1 / 2)} V_{16}+C_{16}^{(1 / 2,1 / 2)} C_{16}\right)-\bar{C}_{8}\left(V_{16}^{(1 / 2,1 / 2)} C_{16}+C_{16}^{(1 / 2,1 / 2)} V_{16}\right)\right\} .
\end{aligned}
$$

For generic points in the moduli space, the massless spectrum of this model is

- the nine-dimensional gravity multiplet: graviton $G_{\mu v}$, anti-symmetric tensor $B_{\mu v}$, dilaton $\phi$;

- the gauge bosons transforming in the adjoint representation of $S O(16) \times S O(14) \times U(1)^{3}$;

- a spinor transforming in the $(\mathbf{1 2 8}, \mathbf{1})$ of $S O(16) \times S O(14)$.

As well in Model I, there are some special points in the moduli space. One of them is $t_{1}=0$, which corresponds to the no Wilson line case in section 几, and the additional massless states are

- two vectors transforming in the $(1,14)$ of $S O(16) \times S O(14)$;

- two spinors transforming in the $(\mathbf{1}, \mathbf{6 4})$ of $S O(16) \times S O(14)$.

Of course, the total massless states agree with those in the no Wilson line case. On the other hand, at the point $t_{1}=1$, all the additional massless states are vectors;

- two vectors transforming in the $(\mathbf{1}, 14)$ of $S O(16) \times S O(14)$;

- two vectors transforming in the $(1,64)$ of $S O(16) \times S O(14)$.

\footnotetext{
${ }^{2}$ Although we often omit the Abelian factors, there is the product of $U(1)$ 's so that the total rank of the gauge symmetry is eighteen.
} 
Then, the gauge symmetry is enhanced to $S O(16) \times E_{8}$. Another special points are $t_{1}= \pm 1 / 2$; At the points, we find the following additional massless states:

- two spinors transforming in the $(\mathbf{1}, 14)$ of $S O(16) \times S O(14)$.

So, the gauge symmetry remains $S O(16) \times S O(14)$ and a massless spinor transforms in the $(128,1) \oplus$ $(1,14)$ in $S O(16) \times S O(14)$ at the points $t_{1}= \pm 1 / 2$.

Note that all the above massless states including the additional ones have zero winding numbers. We are not interested in nonzero winding states which are suppressed in the small- $a$ region. In Ref. [25], however, we have found special orbits in the two-dimensional moduli space characterized by $t_{1}$ and $t_{2}$ on which the additional massless states with nonzero winding numbers appear.

\subsection{The cosmological constants}

The cosmological constant is defined as the integral of the partition function over the fundamental region of the modular group:

$$
\Lambda^{(D)}=-\frac{1}{2}\left(4 \pi^{2} \alpha^{\prime}\right)^{-D / 2} \int_{\mathscr{F}} \frac{d^{2} \tau}{\tau_{2}^{2}} Z^{(D)} .
$$

If the partition function is written as the form $(\mathbb{L}-\mathbb{C})$, we can evaluate the cosmological constant and derive the formula $(\mathbb{L} \cdot \mathbb{6})$ in the small- $a$ region. The procedure of the calculation remains the same even if the partition function is deformed by constant backgrounds.

In Model I with one Wilson line, the cosmological constant in the small-a region is written as the following function of the moduli, up to exponentially suppressed terms:

$$
\Lambda_{\text {Model I }}^{(9)}\left(t_{1}, t_{2}\right) \simeq C_{0}\left(\sqrt{\alpha^{\prime}} t_{2}\right)^{-9} 8\left\{(224-220)+2(16-14) \cos \left(2 \pi t_{1}\right)\right\},
$$

where $C_{0}$ is a positive constant. Note that 224 is the degree of freedom of the bi-fundamental representation of $S O(16) \times S O(14)$ and 220 is that of the adjoint representation of $S O(16) \times S O(14) \times$ $U(1)$ plus the vector of $S O(8)$. We see that $\Lambda_{I}^{(9)}$ is already invariant under the shift $t_{1} \rightarrow t_{1}+1$, which reflects that the massless spectrum at $t_{1}=0$ is the same as that at $t_{1}=1$.

In Model II, the cosmological constant in the small- $a$ region is

$$
\Lambda_{\text {Model II }}^{(9)}\left(t_{1}, t_{2}\right) \simeq C_{0}\left(\sqrt{\alpha^{\prime}} t_{2}\right)^{-9} 8\left\{\left(2^{7}-220\right)-2 \cdot 14 \cos \left(2 \pi t_{1}\right)+2 \cdot 2^{6} \cos \left(i t_{1}\right)\right\} .
$$

Note that $2^{7}$ and $2^{6}$ are the degrees of freedom of the spinor representations of $S O(16)$ and $S O(14)$ respectively.

Figure 1 shows the cosmological constants of Model I and Model II as the one-loop effective potential of $t_{1}$. Note that the gauge symmetry is enhanced at the extrema of the potentials. In particular, in Model I, $t_{1}$ is stabilized at the points $t_{1}= \pm 1 / 2$, where the gauge symmetry is enhanced to $S O(18) \times S O(14)$. Taking account into that the cosmological constant is suppressed at the points, these minima seem to be good vacua. In Ref. [26], however, we deform the interpolating models by the complete set of Wilson lines and it turns out that the other Wilson lines have tachyonic directions at the points. Therefore, the points $t_{1}= \pm 1 / 2$ are, in fact, saddle points and the vacua decay to the other points where the cosmological constant is negative. 

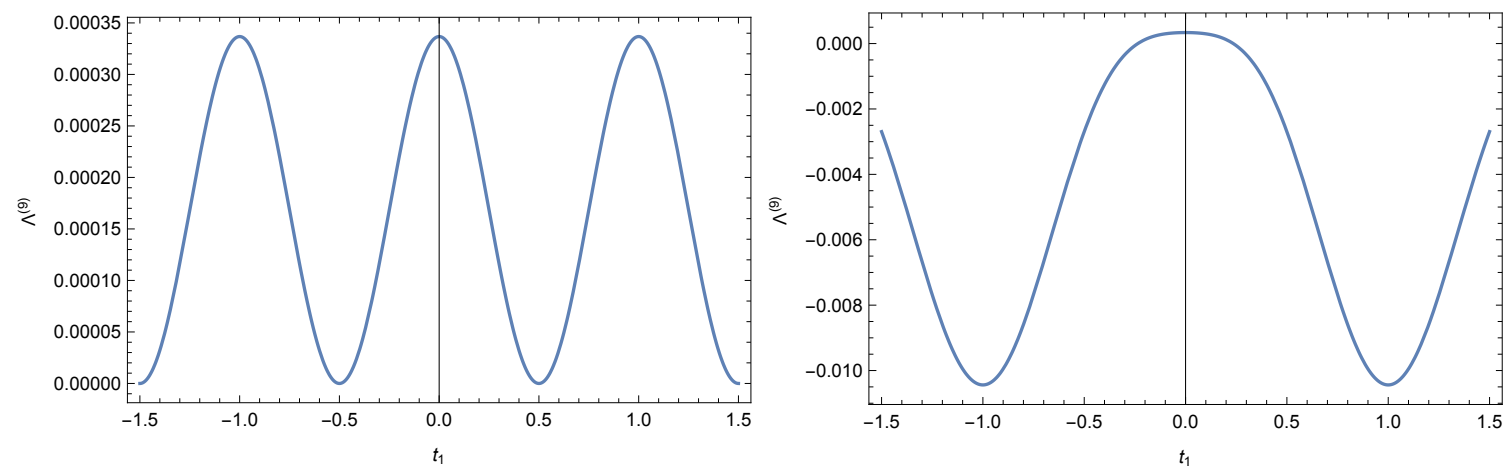

Figure 1: The left and right figures show the cosmological constants $\Lambda^{(9)}$ with $a_{0}=1$ of Model I and Model II respectively, in units of $\alpha^{\prime}=1$, up to exponentially suppressed terms. The cosmological constants decay albegraically as $t_{2} \rightarrow \infty$.

\section{Summary}

We have constructed two 9D interpolating models between the 10D supersymmetric heterotic models and the 10D $S O(16) \times S O(16)$ model and deformed them by one Wilson line. The massless spectra of the models depend on values of the moduli and there are special points in the moduli space where the additional massless states appear. We have listed the special points and the corresponding massless spectra. We have evaluated the cosmological constants as functions of the moduli in the small- $a$ region. The cosmological constant of Model I is exponentially suppressed and the Wilson line is stabilized when the gauge symmetry is enhanced to $S O(18) \times S O(14)$.

\section{Acknowledgments}

I thank H. Itoyama for valuable discussions and helpful comments. I thank the organizers of Conference on Recent Developments in Strings and Gravity of the Corfu Summer Institute 2019 for the opportunity to present recent our works.

\section{References}

[1] P. Candelas, G. T. Horowitz, A. Strominger and E. Witten, "Vacuum Configurations for Superstrings," Nucl. Phys. B 258, 46 (1985).

[2] L. J. Dixon, J. A. Harvey, C. Vafa and E. Witten, “Strings on Orbifolds,” Nucl. Phys. B 261, 678 (1985).

[3] L. J. Dixon, J. A. Harvey, C. Vafa and E. Witten, “Strings on Orbifolds. 2.," Nucl. Phys. B 274, 285 (1986).

[4] L. J. Dixon and J. A. Harvey, "String Theories in Ten-Dimensions Without Space-Time Supersymmetry,” Nucl. Phys. B 274, 93 (1986).

[5] L. Alvarez-Gaume, P. H. Ginsparg, G. W. Moore and C. Vafa, "An O(16) x O(16) Heterotic String," Phys. Lett. B 171, 155 (1986). 
[6] H. Itoyama and T. R. Taylor, "Supersymmetry Restoration in the Compactified O(16) x O(16)-prime Heterotic String Theory,” Phys. Lett. B 186, 129 (1987).

[7] R. Rohm, "Spontaneous Supersymmetry Breaking in Supersymmetric String Theories," Nucl. Phys. B 237, 553 (1984).

[8] C. Kounnas and B. Rostand, "Coordinate Dependent Compactifications and Discrete Symmetries," Nucl. Phys. B 341, 641 (1990).

[9] J. Scherk and J. H. Schwarz, "Spontaneous Breaking of Supersymmetry Through Dimensional Reduction,” Phys. Lett. 82B, 60 (1979).

[10] J. D. Blum and K. R. Dienes, "Duality without supersymmetry: The Case of the $S O(16) x \operatorname{SO}(16)$ string,” Phys. Lett. B 414, 260 (1997) [hep-th/9707148].

[11] H. Itoyama and T. R. Taylor, "Small Cosmological Constant in String Models," FERMILAB-CONF-87-129-T, Proceedings of International Europhysics Conference on High-energy Physics, 25 June-1 July 1987. Uppsala, Sweden (C87-06-25).

[12] C. Kounnas and H. Partouche, "Stringy N = 1 super no-scale models," PoS PLANCK 2015, 070 (2015) [arXiv:1511.02709 [hep-th]].

[13] C. Kounnas and H. Partouche, "Super no-scale models in string theory," Nucl. Phys. B 913, 593 (2016) [arXiv:1607.01767 [hep-th]].

[14] C. Kounnas and H. Partouche, " $\mathscr{N}=2 \rightarrow 0$ super no-scale models and moduli quantum stability," Nucl. Phys. B 919, 41 (2017) [arXiv:1701.00545 [hep-th]].

[15] T. Coudarchet, C. Fleming and H. Partouche, "Quantum no-scale regimes in string theory," Nucl. Phys. B 930, 235 (2018) [arXiv:1711.09122 [hep-th]].

[16] T. Coudarchet and H. Partouche, "Quantum no-scale regimes and moduli dynamics," Nucl. Phys. B 933, 134 (2018) [arXiv:1804.00466 [hep-th]].

[17] H. Partouche, "Quantum no-scale regimes and string moduli,” Universe 4, no. 11, 123 (2018) [arXiv:1809.03572 [hep-th]].

[18] I. Florakis and J. Rizos, "Chiral Heterotic Strings with Positive Cosmological Constant," Nucl. Phys. B 913, 495 (2016) [arXiv:1608.04582 [hep-th]].

[19] S. Abel, K. R. Dienes and E. Mavroudi, "Towards a nonsupersymmetric string phenomenology," Phys. Rev. D 91, no. 12, 126014 (2015) [arXiv:1502.03087 [hep-th]].

[20] B. Aaronson, S. Abel and E. Mavroudi, "Interpolations from supersymmetric to nonsupersymmetric strings and their properties," Phys. Rev. D 95, no. 10, 106001 (2017) [arXiv:1612.05742 [hep-th]].

[21] S. Abel and R. J. Stewart, "Exponential suppression of the cosmological constant in nonsupersymmetric string vacua at two loops and beyond,” Phys. Rev. D 96, no. 10, 106013 (2017) [arXiv:1701.06629 [hep-th]].

[22] S. Abel, K. R. Dienes and E. Mavroudi, "GUT precursors and entwined SUSY: The phenomenology of stable nonsupersymmetric strings,” Phys. Rev. D 97, no. 12, 126017 (2018) [arXiv:1712.06894 [hep-ph]].

[23] K. S. Narain, "New Heterotic String Theories in Uncompactified Dimensions < 10," Phys. Lett. 169B, 41 (1986).

[24] K. S. Narain, M. H. Sarmadi and E. Witten, "A Note on Toroidal Compactification of Heterotic String Theory," Nucl. Phys. B 279, 369 (1987). 
[25] H. Itoyama and S. Nakajima, "Exponentially suppressed cosmological constant with enhanced gauge symmetry in heterotic interpolating models," Prog Theor Exp Phys (2019) [arXiv:1905.10745 [hep-th]].

[26] H. Itoyama and S. Nakajima, "Stability, enhanced gauge symmetry and suppressed cosmological constant in 9D heterotic interpolating models," arXiv:2003.11217 [hep-th]. 